\title{
Beyond reporting: the MJA takes an active role in improving health care
}

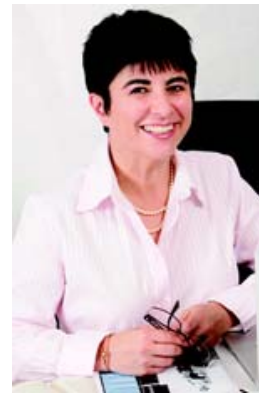

Annette Katelaris

Editor

akatelaris@mja.com.au doi: 10.5694/mjal2.c0716

- MIJA Poocast $\mathrm{n}$ this issue, we report a significant study by Runciman and colleagues that was designed to measure how well we deliver "appropriate care" to patients in Australia (page 100). The researchers were aiming to reproduce a landmark 2003 study that found that only $55 \%$ of patients in the United States received "recommended care". While the methods used by the American and Australian studies were slightly different, the findings are essentially the same - that almost half of patients are not receiving appropriate care. Defining just what is appropriate care was a major challenge for both research groups. The results are sure to make many clinicians bristle and wonder how we enjoy such excellent health outcomes if we fail to deliver appropriate care nearly half the time.

In an editorial, Scott and Del Mar clearly articulate the limitations of the study and why the results may be called into question (page 67). Ackermann defends the care delivered by general practitioners, especially when the significant barriers that obstruct delivery of appropriate care, including limitations in physical and financial access to care, are considered (page 76). He says general practice is already well advanced in using clinical audits to facilitate improved care delivery, but argues that there is more to the delivery of appropriate care than simply ticking off a list of indicators.

Runciman et al's study and their accompanying perspective (page 78) demonstrate the difficulty of their task. They approached more than 15000 people and the health care providers of 2638 eligible participants to recruit their sample. Ethics approval was obtained from more than 220 groups. A key challenge the researchers faced was defining suitable indicators against which to measure best practice. They looked for guidelines to adopt, but found many were duplicated, out of date or non-existent for particular clinical conditions. More often than not, they needed to use consensus-based guidelines developed by small groups of experts. This highlights a challenge that practitioners regularly face - how to access reliable, updated and credible information about appropriate care, and how to make clinical decisions in the absence of this information.

In their perspective article, Runciman et al suggest a way to achieve national agreement on clinical standards - a problem that has also been on the MJA's agenda for some time. We are already working with the Cancer Council Australia to deliver a "wiki" guideline tool on our website. We plan to work with Runciman et al's group to convene a series of disease-specific summits, beginning with the 22 conditions they studied, to better define clinical indicators and standards. We will invite all the main stakeholders in each field, including consumers, and, starting with the evidence, aim to seek a consensus view. These findings will be incorporated into the wiki platform, overseen by nominated experts. This will provide a dynamic, centralised and inclusive platform - openly available to all to contribute to and use - that will help empower clinicians to deliver the best care.

Runciman et al propose that these findings then be integrated into clinical tools to guide and inform care. General practice groups have already attempted to provide these tools. However, tools are only as good as the data that inform them. It is time to improve this information and systematically gather data on what care is routinely delivered. A robust, flexible and reliable information technology platform is clearly essential. How this information is used will no doubt determine whether or not doctors will be willing to engage in this process.

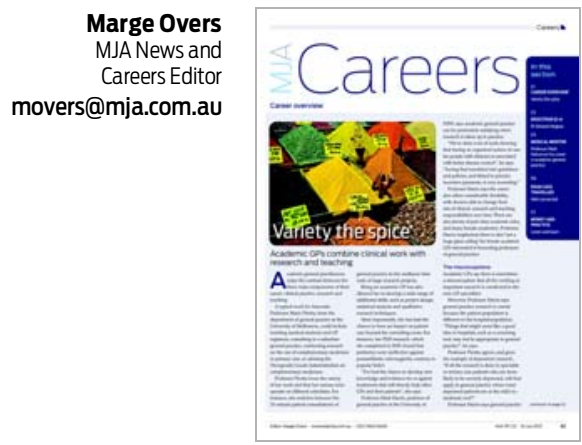

Careers follows $p 122$

\section{Variety is the spice}

Academic general practitioners say they have the best of three worlds: clinical practice, research and teaching. This issue's Career Overview focuses on the rewards of that variety and the importance of GPs being involved in research that can have a direct impact on patient care (page C1). Medical mentor Professor Mark Nelson retraces his career in general practice, academia and research and his balanced lifestyle in
Tasmania (page C5). In Road Less Travelled, we meet Dr Mike Cadogan, who works full-time in emergency medicine and has established an emergency medicine blog that attracts 20000 visitors a day (page C6). Somehow he also finds time to be a physician for a rugby union team and to run a VIP health service. Finally, Money and Practice sets out practical ways to ensure a patient complaint does not end up in court (page C7). 
Editor's choice 